\title{
Premessa all'edizione cinese del Discorso sulla dignità umana di Pico della Mirandola
}

\author{
Pier Cesare Bori, Univerity of Bologna
}

\begin{abstract}
This essay was written as Introduction to the Chinese translation of Pico della Mirandola's Discourse on the Dignity of Man(1486), which is considered the "Manifesto of the Renaissance." The Discourse deals with the vocation of the human creature that, possessing no determinate image, is urged to pursue its own perfection. Such a pursuit begins with moral self-discipline, passes through the familiar, multifarious world of images and fields of knowledge, and strives toward that most lofty goal which defies representation. Pico believes that this paradigm, by virtue of the fact that it is to be found in every tradition, is universal. The Discoursemerits attention today precisely on account of its affirmation that human nature, which is in itself indeterminate and weak, comes alive and obtains its identity through the plurality of human cultures, each representing customs that, though distinct, are (in their essence, structure and function) essentially identical. Hence the possibility of harmony and grounds for "peace" among cultures.
\end{abstract}

1. II Discorso sulla dignità umana di Pico della Mirandola (1463-1494), scritto nel 1486, è considerato il "manifesto del Rinascimento". Le immagini, i colori, i suoni del Rinascimento sono famosi in tutto il mondo. Meno conosciuto tuttavia, al di là degli stereotipi, è questo piccolo capolavoro letterario, che costituisce invece di uno strumento prezioso per penetrare nella mente umanistica. Questo testo infatti - indipendentemente dalla sua origine occasionale (era stato scritto in vista di un evento, la discussione delle Novecento tesi di Pico in un'assemblea romana, che non si riunì mai) - rappresenta con incomparabile efficacia lo sfondo intellettuale del Rinascimento: l'umanesimo, con la sua fiducia appunto nella natura umana e la sua apertura a differenti discipline, culture, filosofie, religioni.

Breve fu la vita di Pico, e la sua creatività si espresse nell'arco di brevissimi anni, anzi mesi. E tutta questa stagione straordinaria sarebbe durata poco: gli eventi della Riforma e della Controriforma avrebbero cambiato definitamente il volto dell'Europa. Ma l'umanesimo avrebbe comunque segnato 
tutta la storia successiva. Perfino l'impresa di Matteo Ricci (un secolo dopo, ma quanta differenza da Pico) non sarebbe stata possibile senza la passione filologica e lo slancio universalistico che avevano preso impulso da Cusano, Marsilio, Pico, Erasmo.

2. Giovanni Pico della Mirandola nacque il 24 febbraio 1463 nel castello di Mirandola, dei conti di Mirandola e Concordia. La madre, Giulia Boiardo, consapevole dei talenti straordinari del precoce Giovanni, lo volle sottrarre al "mestiere delle armi" comune alla famiglia e alle contese che dividevano i due fratelli Galeotto e Anton Maria, e lo volle avviare agli studi ecclesiastici. Recatosi prima a Bologna, a quattordici anni, per studiare diritto, morta la madre nell'agosto del 1478, andava a Ferrara nel maggio del 1479 per studiarvi filosofia. Qui conosceva anche Girolamo Savonarola e nasceva un rapporto di reciproca stima. A Ferrara coltivò gli "studia humanitatis" con Battista Guarini, e apprese il greco. Nell'ottobre del 1480 si trasferiva a Padova, dove approfondiva la conoscenza di Aristotele e dell'averroismo e veniva introdotto anche alla conoscenza della Cabala ebraica dal filosofo aristotelico Elia del Medigo. Dopo l'estate del 1482 si recò a Pavia, dove si arricchiva con la conoscenza della logica. Nel frattempo maturava il suo incontro con l'umanesimo fiorentino, a partire dall'amicizia con Angelo Poliziano, iniziata in un momento in cui Pico coltivava un interesse verso la produzione poetica. Incontrava naturalmente anche con il grande Marsilio Ficino. Rinunciando all'eredità e risolvendo ogni problema economico con i fratelli, Pico si ritrovò con grandi risorse economiche e libero di perseguire i suoi interessi intellettuali. A Firenze coltivava l'amicizia del Poliziano, di Ficino di Girolamo Benivieni, di Lorenzo de' Medici. Qui ad Elia del Medigo, per la conoscenza delle cose ebraiche, si affiancò Guglielmo Raimondi di Moncada, ovvero Flavio Mitridate, ebreo di origine, personaggio complesso e difficile, ma validissimo traduttore. Con un soggiorno a Parigi, alla Sorbona, tra il luglio del 1485 e l'inizio dell'anno successivo, si completava una formazione che aveva consentito a Pico, più di ogni altro intellettuale nel suo tempo, la conoscenza delle più importanti correnti culturali e dei protagonisti più significativi della cultura europea.

3. Il $1486 \mathrm{fu}$ per il giovane Pico della Mirandola un anno straordinario. Nel marzo, a 23 anni, tornò da Parigi a Firenze, tra i suoi amici Lorenzo de' Medici, Poliziano, Marsilio Ficino. L'otto maggio partì per Roma. Due giorni dopo ad Arezzo tentava senza successo di rapire Margherita, moglie di Giuliano de' Medici, causando molto scandalo. Riparatosi a Fratta, tra Perugia e Todi, si apriva per lui un periodo di nascondimento eccezionalmente fecondo. Dopo alcuni mesi di silenzio, a partire dal settembre dello stesso anno, la corrispondenza ricomincia a ridarci notizia di lui. Tre lettere di quel periodo sono importanti, anche per la comprensione del suo Discorso. 
Anzitutto Pico scriveva a Marsilio Ficino parlandogli con entusiasmo dei suoi studi linguistici, dei sapienti caldei, del suo amore per Plotino. Si può pensare che risalga a questo periodo la prima, parziale redazione (testimoniata dal Codice palatino 885), del Discorso e che risalga anche, insieme a questo, il disegno di un convegno a Roma. Si sarebbe trattato di un ampio consesso di teologi, filosofi, personalità della Chiesa, in cui Pico avrebbe riscattato il suo onore e si sarebbe ricoperto di gloria proponendo alla discussione un insieme di novecento proposizioni (Tesi) in cui si avrebbe compendiato il sapere del suo tempo. II Discorso sarebbe stato allora l'introduzione alla disputa romana.

II 15 ottobre, scriveva ad Andrea Corneo una lunga lettera. Parlava dei fatti del maggio precedente. Si doleva di aver peccato, non si difendeva. Altri avrebbero voluto scusarlo di quello di cui egli non si scusava affatto. Ma difendeva la vocazione intellettuale e filosofica, anche nella sua funzione sociale.

Infine il 10 novembre informava un ignoto amico che le sue tesi sono cresciute sino a 900. Al Discorso si aggiungeva un'ultima sezione ispirata al testo evangelico: "Vi do la mia pace, vi do la mia pace, vi lascio la pace" (Gv 14,27-31a). Pico sentiva con commozione che la sua impresa avrebbe potuto contribuire alla pacificazione di un mondo diviso.

Entusiasmo ermeneutico, impresa filosofico-teologica (senza opposizione tra le due discipline), al servizio della pace interiore ed esterna: questi allora sono i temi originari di questo testo, elaborato in solitudine. A questi venne ad aggiungersi, forse quando Pico era già a Roma, un quarto tema: la difesa della propria impresa contro le obiezioni e le accuse che Pico prevedeva certo ci sarebbero state, ma certo non così aspre e distruttive.

4. Si recava dunque a Roma dove, il 7 dicembre le Tesi venivano pubblicate. Le reazioni furono deludenti. Papa Innocenzo VIII all'inizio del 1487 vietò la pubblica discussione. II 29 febbraio 1487 venne nominata una commissione di vescovi, teologi, religiosi che si riunì tra il 2 e il 13 marzo. Pico partecipò alle sedute, ma solo per cinque giorni. Sette sue tesi vennero condannate, altre sei censurate. Nella primavera del 1487 Pico preparò rapidamente una Apologia e la pubblicò (il Discorso apparve invece solo nel 1496, dopo la sua morte pubblicato dal nipote Giovanfrancesco insieme ad altri scritti). Attaccato e condannato per alcune sue tesi, Pico rispose con la sua Apologia. Il testo è importante, perché Pico vi si difende distinguendo diversi livelli di certezza. Un conto è il credo: gli articoli di fede derivati direttamente dalla Bibbia e dai primi quattro concili e anche (su un gradino inferiore) le formulazioni dogmatiche dei concili successivi, e un conto sono le opinioni dei Padri della Chiesa e della teologia. Le opinioni possono essere vere, false, probabili, verosimili, ma contrastarle non è eretico. Altro è la fede, altro è l'opinione. Rinviato all'Inquisizione, il 31 luglio sottoscrisse un atto di sottomissione 
all'autorità ecclesiastica. Le Tesi dovevano essere bruciate. Offeso, amareggiato, Pico abbandonò Roma e si recò in Francia; nel viaggio venne arrestato e scortato a Parigi. Protetto dal re, accolto a Vincennes, poté ritornare a Firenze nell'aprile del 1488. Alessandro VI lo riconcilierà presto con la Chiesa cattolica e cancellerà la sua condanna.

Gli ultimi anni di Pico furono segnati da una produzione intensa ma anche da un certo ritorno a posizioni meno originali. Nell'estate del 1489 godendo di un felice raccoglimento a Fiesole, attese a un commento ai Salmi e al primo capitolo della Genesi. Scriveva nello stesso periodo il Sull'ente e sull'uno, dedicato ad Angelo Poliziano, che avrebbe circolato manoscritto, frammento di una grande opera che avrebbe dovuto dimostrare la "concordia" di Aristotele con Platone. Attendeva anche ad un'opera assai vasta, le Dispute contro l'astrologia divinatrice, che sarà pubblicata, incompleta, dal nipote nel 1496. Pico vi distingue tra astronomia e astrologia, contestando la validità di quest'ultima, contro la voga del tempo, favorita anche da altri studiosi come Marsilio Ficino. Giovanni Pico morì dopo breve malattia, per una febbre, il 17 novembre 1494, assistito fra gli altri da fra' Girolamo Savonarola, cui era stato molto vicino, negli ultimi anni della sua breve vita.

5. La figura di Pico, sin dalla sua morte prematura, non ha cessato di affascinare la fantasia popolare, e di attirare l'attenzione degli studiosi. Negli ultimi decenni si sono tenuti importanti convegni, nuove edizioni e traduzioni delle sue opere sono apparse, le sue fonti sono state indagate in profondità. In tutto questo fermento, permane tuttavia, ormai da decenni, la divaricazione fra due atteggiamenti interpretativi intorno a Pico.

La tendenza dominante dell'interpretazione pichiana - da Jacob Burckhardt, con la sua classica opera sul Rinascimento in Italia, attraverso Giovanni Gentile, Eugenio Garin (il maggior studioso di Pico), Ernst Cassirer, Paul Oskar Kristeller, sino agli ultimi contributi sulla cultura magica, astrologica, esoterica, cabbalistica, sincretistica di Pico - la tendenza dominante insiste su Pico come innovatore, rappresentante eminente della modernità, con il suo individualismo e la sua esaltazione della libertà. Un'altra tendenza vede in Pico - e nel suo Discorso la continuità con la tradizione teologica, dalla Bibbia, ai Padri, alla teologia scolastica. Fra i rappresentanti di questa tendenza, Henri De Lubac si distingue per il genuino pathos e per l'alto grado di erudizione (e fra gli altri, eccelle il lavoro di Di Napoli).

Gli anni più recenti hanno visto aumentare contributi molto importanti dedicati allo studio e edizione delle singole opere, fra cui la fondamentale edizione curata da Francesco Bausi, e quella che ho curato con Francesco Borghesi, Michael Papio e Massimo Riva per la Cambridge University Press. Gli studi ebraici e islamici di Pico sono più noti e indagati. 
6. Per nostra parte, la conoscenza di Pico si inserisce in una ricerca in cui abbiamo sempre privilegiato gli esponenti di una ricerca religiosa certo, ma universalistica e pluralistica. Così è stato all'inizio del Pico-Project con la Brown University nel 1997 (con Massimo Riva, Mike Papio, Saverio Marchignoli, Dino Buzzetti, Francesco Borghesi e altri). La premessa tuttora leggibile in rete diceva:

II Discorso sulla dignità dell'uomo di Pico della Mirandola (1463-1494) è considerato il manifesto del Rinascimento. Scritto nel 1486, contiene infatti l'esaltazione della creatura umana, come creatura libera e capace di conoscere e dominare la realtà intera. Ancor più di questo però il Discorso parla del compito della creatura umana: questa, priva di immagine predeterminata, deve perseguire la propria compiutezza con un percorso che muove dall'autodisciplina morale, attraversa la pluralità delle immagini e dei saperi, e tende alla meta più alta, non rappresentabile. Pico della Mirandola ritiene che questo paradigma di sviluppo dell'esistenza sia universale, perché rintracciabile in tutte le tradizioni. L'interesse attuale del Discorso è proprio nella sua affermazione che la natura umana, indeterminata e debole di per sé, si realizza e si identifica attraverso la realtà molteplice delle culture umane: ogni cultura costituisce una via diversa, ma nella sua essenza, funzione e struttura, identica. Di qui anche la possibilità della concordia e il fondamento della pace, tra le culture. (Progetto Picol Pico Project)

7. In un contributo più organico, alcuni anni fa, dal titolo già significativo, "Pluralità delle vie," approfondivo le principali chiavi di lettura: anzitutto la difesa della libera vocazione intellettuale e la fiducia ermeneutica, che sostiene Pico nell'affrontare si può dire per la prima volta insieme letterature e mondi tanto diversi; e quindi la coraggiosa apertura del canone anche di là della Bibbia (ma mai contro di essa) alla ricerca delle fonti di una "filosofia perenne." In terzo luogo il bilinguismo, per cui Pico può parlare della stessa esperienza spirituale in termini biblici (per lui non rinunciabili) $e$ in termini platonici, o comunque filosofici. Di qui la pluralità delle vie, che attraverso un modello tripartito di evoluzione morale, intellettuale, spirituale, conducono comunque tutte ad un esito unitario; e infine quel che si può dire la via della bellezza, la "teologia poetica" che gli consente di affermare che in ogni scuola c'è qualcosa di "insigne" (riprendendo Cicerone), e di articolare il discorso secondo i diversi autori e di apprezzare lo stile di ogni autore.

8. Volendo andar ancor oltre si potrebbe tentare di riassumere le premesse su cui si basa la visione di Pico, per quel che possiamo ricostruire dalla frammentarietà dei suoi scritti (com'è noto, le Tesi sono pure enunciazioni, prive del corpo argomentativo). Anzitutto il paradigma genealogico e l'unità iniziale del 
sapere umano. Alla base sta certamente Marsilio Ficino, che con il suo straordinario lavoro, anzitutto di traduzione, volto alla valorizzazione degli antichi misteri e della primitiva teologia, aveva preparato il terreno a Pico. Nel Discorso (soprattutto nella prima redazione) e nelle sue Tesi Pico sembra adombrare una genealogia originale, in cui i "caldei", hanno un ruolo fondamentale, perché connessi a Zoroastro. Parallelamente c'è la fonte ebraica, meglio rappresenta dalla Cabbala, che Pico rivendica il merito di avere per primo valorizzato. Si vuol suggerire che, via via che si retrocede verso la sapienza antica, ci si avvicina ad una filosofia perenne che contiene in sé $i$ fondamenti di un'unità di pensiero smarrita nel corso della storia del pensiero umano. Le fasi più antiche di questa filosofia sono avvolte da un linguaggio simbolico e poetico, una "teologia poetica"; ma Pico crede di poter mostrare le corrispondenze che vi sono tra le diverse formulazioni di questo pensiero: soprattutto zoroastrismo e Cabbala, poi sapienza egizia e orfica.

In secondo luogo la com-presenza degli esseri. Al di là della dimostrazione della convergenza verso le origini comuni, Pico propone, al centro delle sue Tesi, un fondamento filosofico originale della concordia tra le divergenti scuole di pensiero. Si tratta delle "settantuno tesi paradossali secondo la propria opinione, introducenti nuove dottrine nella filosofia": è la sua "Nuova filosofia." Per quel che possiamo comprendere, l'Autore concepisce e propone diversi stati o livelli dell'essere, l'ultimo o primo di questi è quel che chiama essere "uniale", che stabilisce la fondamentale correlazione tra tutti gli esseri. II principio fondamentale è che sebbene, come è tradizione teologica, le gerarchie divine siano distinte, si deve tuttavia intendere che tutto è in tutto a suo modo. II riferimento a Nicola da Cusa viene spontaneo, ma non è certo. Sicuramente la formula "tutto in tutto" è di antica ascendenza. Steve Farmer ne indica l'origine nel neoplatonismo, rispondendo questo principio a quel che Farmer ritiene esigenze sincretistiche. Pico tuttavia fa uso di questo principio dell'unicità ultima del reale sul piano dell'ermeneutica teologica e sul piano metafisico con tale coerenza teoretica di rendere ingiustificata l'accusa di sincretismo. II progetto di concordia filosofica nel senso più vasto, quale era stato quello delle Tesi, troverebbe qui, secondo questa lettura, una giustificazione più profonda che nel paradigma dell'analogia e della partecipazione, dell'emanazione e della gerarchia degli esseri, in cui l'unità essenzialistica dell'essere è acquisita attraverso una dialettica intellettualistica.

Nasce di qui quella intertestualità che si può dire infinita, della quale si è già detto, ma anche anche l'intuizione di una possibilità di intervenire sulla natura in forza del legame che unisce tutti gli esseri ("simpatia" universale) e grazie conoscenza delle leggi che governano la natura: il motto di Francesco Bacone, secondo cui l'uomo non comanda alla natura se non obbedendole, compare già nel Discorso. II contesto è quello, tipicamente rinascimentale, della 
rivalutazione della "magia" come espressione dell'antico sapere originario, ma è corretto riconoscere qui una tensione verso un sapere che diventi anche tecnica al servizio dell'uomo.

Infine, l'unità escatologica, personale e comune. L'impossibilità di definire l'immagine divina getta luce sulla prima pagina del Discorso: anche l'uomo è "privo di immagine definita." II destino, individuale e comune dell'umanità, è nel ritorno all'unità con un Assoluto del quale solo "il silenzio è lode". In un passo del breve trattato "Sull'ente e sull'uno" (cap. 5), a proposito dell'oscurità in cui Dio abita, Pico cita un passo straordinario di Dionigi Areopagita (Teologia mistica 5) che così parla della divinità: "Non è verità, né regno, né sapienza, né uno, né unità, né divinità, né bontà, né spirito...Nella divinità non c'è nessun discorso, né nome, né scienza, né tenebre, né luce, né errore, né verità, né è possibile di lei alcuna affermazione o negazione alcuna" (Dyonisius, De mystica theologia 149-150).

Le premesse storiche per la tesi pichiane sulla genealogia della sapienza erano troppo deboli. L'amore per il linguaggio non significa per lui la stessa cosa che per il filologo Lorenzo Valla. Le connessioni intertestuali di Pico sono ancora fragilmente sorrette dall'allegorismo dell'antica esegesi. II fascino dell'esoterico e del magico impregna in maniera per noi fastidiosa le ultime pagine. La cultura retorica e il latino umanistico rendono Pico perfino più difficile a leggersi che la stessa scolastica medievale.

E tuttavia è importante cogliere anche quanto di teoreticamente rilevante moderno? attuale? (si possono ancora usare questi aggettivi in questa età posthegeliana e post-storicista?) - c'è nella pagine di Pico: pensiamo al tema dell' "età assiale," proposto da Karl Jaspers e tuttora accolto dalla ricerca. Pensiamo a Leibniz, che oggi ottiene sempre maggiore attenzione. Pensiamo al superamento dell'opposizione tra sapere filosofico sapere religioso, recentemente invocato da J. Habermas. Pensiamo, a proposito della compresenza degli esseri, a quell' "unicità dell'esistenza" che la filosofia religiosa araba medievale proponeva e che un grande comparatista, Toshiko Izutsu (Sufism and Taoism) vedeva come anche come terreno comune delle grandi tradizioni.

9. Ed ecco l'edizione cinese, frutto di un'esperienza di lavoro in comune già come tale, crediamo, molto interessante e potenzialmente aperta ad altri risultati. Le considerazioni che precedono potranno interessare anche il lettore in Cina, crediamo, non siamo, come si sarà compreso, tra i sostenitori di invalicabili differenze tra le culture, tanto meno di inevitabili conflitti. Ma in particolare potrà interessare questo lettore, come già si accennava, di incontrare, nel momento della sua formazione, quella cultura umanistica che avrebbe reso possibile il più positivo scambio tra cultura europea e quella cinese, con Matteo Ricci e gli altri compagni. 
Più in generale, se si considera che la cultura confuciana presenta lo stesso tratto fondamentale: "lo tramando, non fabbrico: stimo e amo gli antichi" (Lun Yü, VII, 9), sarà facile apprezzare in Cina - che pure, in termini diversi, ha conosciuto un secolo fa un suo "Rinascimento" (Hu Shi) - l'attenzione ai classici e il desiderio di renderli vivi e attuali, al servizio del presente.

E infine, la fondamentale fiducia antropologica, l'idea di un dovere-diritto umano di valorizzare liberamente le proprie potenzialità $e$ al tempo stesso il bisogno di armonia e di pace... tutto questo troverà sicuramente qui un eco.

Per non dire dell'appello alla bellezza, come ultima istanza di verità.

Pechino, 20 aprile 2010

\section{Works Cited}

Bori, Pier C, and Saverio Marchignoli. Pluralità delle vie: alle origini del Discorso sulla Dignità Umana di Pico Della Mirandola. Milano: Feltrinelli, 2000. Print.

Bori, Pier C., "The Modern Search for Universalism in Western Culture." Culture,

Religion and the Problem of Universalism. Ed. Y. Shibata. Yokohama: Meiji Gakuin University, 1999. 19-30. Print

Cassirer, Ernst. "Giovanni Pico Della Mirandola: a Study in the History of Renaissance Ideas." Journal of the History of Ideas. 3.3 (1942): 319-346. Print.

Confucio, and Alberto Castellani. I Dialoghi di Confucio (Lun Yú). Firenze: Sansoni, 1949. Print.

Di Napoli, G. Giovanni Pico della Mirandola e la problematica dottrinale del suo tempo. Roma: Desclée, 1965. Print.

Dionysius, Areopagita, Pseudo. Günther Heil, and Adolf M. Ritter. Corpus 
Dionysiacum / II, De coelesti hierarchia. De ecclesiastica hierarchia. De mystica theologia. Epistulae. Berlin: De Gruyter, 1991. Print.

Farmer, Steve A. and Pico della Mirandola, G. Syncretism in the West: Pico's 900 Theses (1486): the Evolution of Traditional, Religious, and Philosophical Systems: with Text, Translation, and Commentary. Tempe, Ariz: Medieval \& Renaissance Texts \& Studies 1998. Print.

Garin, Eugenio. Giovanni Pico della Mirandola. Vita $e$ dottrina. Firenze: F. Le Monnier, 1937. Print.

Gentile, Giovanni. "II concetto dell'uomo nel Rinascimento." Giornale Storico della Letteratura Italiana 67 (1916): 17-69.

Izutsu, Toshihiko. Sufism and Taoism: A Comparative Study of Key Philosophical Concepts. Berkeley: University of California Press, 1984. Print.

Lubac, Henri, and Georges Chantraine. Histoire Et Esprit: L'intelligence De L'écriture D'après Origène. Paris: Éd. du Cerf, 2002. Print.

---. Pic De La Mirandole: Études Et Discussions. Paris: Aubier Montaigne, 1974. Print.

Wirszubski, Chaim. Pico Della Mirandola's Encounter with Jewish Mysticism. Cambridge, Mass: Harvard University Press, 1989. Print. 
Pico della Mirandola, G., Francesco Borghesi, Michael Papio, and Massimo Riva. Oration on the Dignity of Man: A New Translation and Commentary. New York: Cambridge University Press, 2012. Print.

Pico della Mirandola, G., and Francesco Bausi. Discorso Sulla Dignità Dell'uomo. Parma: Fondazione Pietro Bembo, 2003. Print.

Pico della Mirandola, G., Pier C. Bori, Massimo Riva, and Michael Papio. Progetto Pico: Pico Project: De Hominis Dignitate: un progetto di collaborazione tra Università degli Studi di Bologna e Brown University. Providence, RI: Brown University Dept. Of Italian Studies, 1999. Internet resource.

Pico della Mirandola, G., and Francesco Bausi. Opere Complete. Roma: Lexis Progetti Editoriali, 2000. CD for computer.

Progetto Pico/Pico Project. Web. 12 Dec. 2012. <www.brown.edu/Departments/ Italian_Studies/pico>.

Pico della Mirandola, G., Eugenio Garin. De Hominis Dignitate; Heptaplus; De Ente Et Uno; E Scritti Vari. Firenze: Vallecchi, 1942. Print. 